\title{
STK25 regulates oxidative capacity and metabolic efficiency in adipose tissue
}

\author{
Silva Sütt1,*, Emmelie Cansby1,*, Alexandra Paul2, Manoj Amrutkar³, Esther Nuñez-Durán', Nagaraj M Kulkarni1, \\ Marcus StåhIman ${ }^{4}$, Jan Borén ${ }^{4}$, Jurga Laurencikiene ${ }^{5}$, Brian W Howell, ${ }^{6}$ Sven Enerbäck7 and Margit Mahlapuu1 \\ 1'Lundberg Laboratory for Diabetes Research, Department of Molecular and Clinical Medicine, Institute of Medicine, University of Gothenburg, \\ Sahlgrenska University Hospital, Gothenburg, Sweden \\ 2Department of Biology and Biological Engineering, Division of Chemical Biology, Chalmers University of Technology, Gothenburg, Sweden \\ ${ }^{3}$ Department of Hepato-Pancreato-Biliary Surgery, Institute of Clinical Medicine, University of Oslo, Oslo, Norway \\ ${ }^{4}$ Department of Molecular and Clinical Medicine/Wallenberg Laboratory, Institute of Medicine, University of Gothenburg, Sahlgrenska University \\ Hospital, Gothenburg, Sweden \\ ${ }_{5}^{5}$ Lipid Laboratory, Department of Medicine Huddinge, Karolinska Institutet, Stockholm, Sweden \\ ${ }^{6}$ Department of Neuroscience and Physiology, State University of New York Upstate Medical University, Syracuse, New York, USA \\ ${ }^{7}$ Department of Medical and Clinical Genetics, Institute of Biomedicine, The Sahlgrenska Academy, University of Gothenburg, Gothenburg, Sweden
}

Correspondence should be addressed to M Mahlapuu: Margit.Mahlapuu@gu.se

*(S Sütt and E Cansby contributed equally to this work)

\section{Abstract}

Whole-body energy homeostasis at over-nutrition critically depends on how well adipose tissue remodels in response to excess calories. We recently identified serine/ threonine protein kinase (STK) 25 as a critical regulator of ectopic lipid storage in non-adipose tissue and systemic insulin resistance in the context of nutritional stress. Here, we investigated the role of STK25 in regulation of adipose tissue dysfunction in mice challenged with a high-fat diet. We found that overexpression of STK25 in high-fat-fed mice resulted in impaired mitochondrial function and aggravated hypertrophy, inflammatory infiltration and fibrosis in adipose depots. Reciprocally, Stk25-knockout mice displayed improved mitochondrial function and were protected against diet-induced excessive fat storage, meta-inflammation and fibrosis in brown and white adipose tissues. Furthermore, in rodent HIB-1B cell line, STK25 depletion resulted in enhanced mitochondrial activity and consequently, reduced lipid droplet size, demonstrating an autonomous action for STK25 within adipocytes. In summary, we provide the first evidence for a key function of STK25 in controlling the metabolic balance of lipid utilization vs lipid storage in brown and white adipose depots, suggesting that repression of STK25 activity offers a potential strategy for establishing healthier adipose tissue in the context of chronic exposure to dietary lipids.
Key Words
- brown adipose tissue
- white adipose tissue
- lipid metabolism
- oxidative stress

Journal of Endocrinology (2018) 238, 187-202

\section{Introduction}

Obesity is an established risk factor for an array of medical problems including insulin resistance, type 2 diabetes, cardiovascular disease, non-alcoholic fatty liver disease (NAFLD) and cancer (Van Gaal et al. 2006, Sjostrom et al. 2009, Anstee et al. 2013, Kusminski et al. 2016).
The mechanism connecting obesity to related diseases is not clearly understood; however, increasing evidence in obese individuals and high-fat diet-fed animal models implicates chronic low-grade inflammation and mitochondrial dysfunction in adipose depots as key 
causative factors in the etiology and progression of obesityrelated pathologies (Xu et al. 2003, Rong et al. 2007, Lumeng \& Saltiel 2011, Heinonen et al. 2015). Obesity has reached epidemic proportions worldwide and currently represents a considerable economic burden for healthcare systems (http://www.who.int/mediacentre/factsheets/ fs311/en/). Hence, a comprehensive understanding of the molecular mechanisms controlling lipid partitioning, meta-inflammation and mitochondrial activity in adipose tissue is critically needed to support the development of effective and safe anti-obesity therapies.

In the search for novel targets that regulate wholebody energy metabolism in the context of nutritional stress and obesity, we recently described serine/threonine protein kinase (STK)25, a member of the sterile 20 (STE20) kinase superfamily (Thompson \& Sahai 2015), as a critical regulator of lipid partitioning and systemic glucose and insulin homeostasis (Nerstedt et al. 2012, Cansby et al. 2013, Amrutkar et al. 2015a,b,2016a,b, Chursa et al. 2017, Nunez-Duran et al. 2017, 2018). We found that STK25-overexpressing transgenic mice display impaired whole-body glucose tolerance and insulin sensitivity compared with WT littermates when fed a high-fat diet (Cansby et al. 2013). Reciprocally, our studies showed that repression of STK25 activity in mice by genetic depletion or antisense oligonucleotide (ASO) treatment protects against high-fat diet-induced glucose intolerance and insulin resistance (Amrutkar et al. 2015a, Nunez-Duran et al. 2018). Furthermore, we observed a markedly accelerated ectopic lipid accumulation, combined with aggravated inflammatory infiltration and fibrosis, in the liver, skeletal muscle and pancreas of high-fat-fed Stk 25 transgenic mice compared with WT littermates (Amrutkar et al. 2015b, Chursa et al. 2017, Nunez-Duran et al. 2017). Reciprocally, protection against diet-induced hepatic and skeletal muscle lipid storage via increased oxidative capacity was seen in Stk25-/- mice (Amrutkar et al. 2015a, 2016a,b). Consistent with these results from mouse models, we also found that STK25 mRNA and protein levels correlate significantly and positively with progression of NAFLD in human liver biopsies, and several common non-linked SNPs in the human STK25 gene are associated with altered liver fat (Amrutkar et al. 2016a,b, Nunez-Duran et al. 2018). Moreover, STK25 mRNA levels are higher in the skeletal muscle of individuals with type 2 diabetes than in healthy volunteers (Nerstedt et al. 2012), supporting a critical role of STK25 in the pathogenesis of metabolic disease in humans.

STK25 is broadly expressed, with high mRNA and protein levels detected both in brown and white adipose tissues (BAT and WAT (Nerstedt et al. 2012, Cansby et al.

() 2018 Society for Endocrinology Published by Bioscientifica Ltd.
Printed in Great Britain
2013, Amrutkar et al. 2015a)); however, the physiological function of STK25 in adipocytes remains elusive. On the basis of our previous findings, which reveal a central role of STK25 in control of lipid accumulation, chronic lowgrade inflammation and fibrosis in key metabolic organs liver, skeletal muscle and pancreas of high-fat-fed mice (Nerstedt et al. 2012, Cansby et al. 2013, Amrutkar et al. 2015a,b, 2016a,b, Chursa et al. 2017, Nunez-Duran et al. 2017,2018 ), we hypothesized that STK25 is also involved in the regulation of adipose tissue dysfunction in connection to obesity. Here, we provide the first evidence for key cell-specific role of STK25 in controlling the mitochondrial function and metabolic balance of lipid utilization vs lipid storage in the BAT and WAT of obese mice, suggesting that STK25 repression offers a strategy for establishing healthier adipose tissue in the context of chronic exposure to dietary lipids.

\section{Material and methods}

Mice

Stk25 transgenic and knockout mice were generated and genotyped as described (Cansby et al. 2013, Matsuki et al. 2013). The genetic background of Stk25 transgenic and knockout line differs (C57BL6/N and C57BL6/J, respectively) and therefore, each line has been compared to their corresponding WT littermates. From the age of 6 weeks, male transgenic and knockout mice, and their corresponding WT littermates, were fed a pelleted high-fat diet (45\% kilocalories from fat; D12451; Research Diets, New Brunswick, NJ, USA). At the age of 24 weeks, the mice were killed after $4 \mathrm{~h}$ of food withdrawal. Interscapular BAT, and subcutaneous and epididymal WAT (sWAT and eWAT) samples were collected for histological analysis and nonlinear microscopy or snap-frozen in liquid nitrogen and stored at $-80^{\circ} \mathrm{C}$ for analysis of protein and gene expression (Supplementary Fig. 1, see section on supplementary data given at the end of this article). All animal experiments were performed after approval from the Local Ethics Committee for Animal Studies at the Administrative Court of Appeals in Gothenburg, Sweden and followed appropriate guidelines.

\section{Histology, immunohistochemistry and immunofluorescence}

BAT and WAT sampleswerefixed with 4\% paraformaldehyde in phosphate buffer, embedded in paraffin, sectioned and stained with hematoxylin and eosin (H\&E) or 
Picrosirius Red. Samples were also embedded in optimal cutting temperature mounting medium and frozen in liquid nitrogen followed by cryosectioning and staining with MitoTracker Red. For immunohistochemistry and immunofluorescence, sections were incubated with primary antibodies followed by incubation with secondary antibodies (Supplementary Table 1).

\section{Biochemical assays}

The fasting plasma leptin and adiponectin levels were assessed using the Mouse Leptin ELISA Kit (Abcam) and the Mouse Adiponectin ELISA Kit (Abcam), respectively. Ceramides were extracted from adipose tissue using the BUME method (Lofgren et al. 2016) and purified using straight phase HPLC coupled to a fraction collector (Homan \& Anderson 1998); the ceramides were then detected and quantified using UPLC-MS/MS as previously described (Amrutkar et al. 2015b). Thiobarbituric acidreactive substance (TBARS) levels were measured in tissue homogenate using the Lipid Peroxidation Assay Kit (Sigma-Aldrich).

\section{Nonlinear and electron microscopy}

A custom-built two-photon excited fluorescence (TPEF) and coherent anti-Stokes Raman scattering (CARS) microscope was used to visualize lipids and active mitochondria within the adipose tissue as described in the Supplementary Materials and methods. Ultrastructural analysis was performed by transmission electron microscopy (LEO 912AB; Omega; Carl Zeiss NTS) (Anderberg et al. 2013).

\section{Lipolytic activity}

Adipocytes were isolated from eWAT (Cansby et al. 2013) and the activity of adipose triacylglycerol lipase (ATGL) and hormone-sensitive lipase (HSL) was determined using $\left[{ }^{3} \mathrm{H}\right]$ triolein (PerkinElmer) or $\left[{ }^{14} \mathrm{C}\right]$-cholesteryl oleate (PerkinElmer) as the substrate, respectively, as previously described (Egan et al. 1992, Reid et al. 2008).

\section{Cell culture, transient transfections and in vitro metabolic assessments}

The HIB-1B cell line (derived from a brown fat tumor and capable of expressing the brown fat-specific mitochondrial uncoupling protein (UCP1), mouse) was cultured and maintained as described (Vernochet et al. 2009).
Cells were transfected with Stk25-specific siRNA (a mixture of seven sets of siRNA against mouse Stk25; s81846; Ambion) or scrambled siRNA (SIC001; SigmaAldrich) using Lipofectamine RNAiMax (Invitrogen). After transfection, cells were differentiated for 5 days as described (Fujimori \& Amano 2011). Samples were collected for analysis of protein and gene expression. Cells were stained with Oil Red $\mathrm{O}$ for lipids or with MitoTracker Red for mitochondria (Supplementary Materials and methods). The $\beta$-oxidation, non-esterified fatty acid (NEFA) uptake, incorporation of [ $\left.{ }^{14} \mathrm{C}\right]$ oleic acid into triacylglycerol (TAG) and lipolysis were measured as described in the Supplementary Materials and methods. To inhibit lipolysis, cells were treated during 5 days with nicotinic acid (NiAc; Sigma-Aldrich).

The mouse embryonic fibroblast cell line 3T3-L1 (CL-173; American Type Culture Collection (ATCC)) was cultured and differentiated into white adipocytes as described (Gustafson \& Smith 2006); transfection with Stk25 or scrambled siRNA was performed as explained above before differentiation was initiated. Cells were stained with Nile Red (Sigma-Aldrich) or processed for immunofluorescence (Supplementary Table 1).

\section{Western blot and quantitative real-time PCR}

Western blotting was performed as described (Nerstedt et al. 2012) (Supplementary Table 1). The anti-STK25 antibody has been validated by using Stk25-/- mice (Amrutkar et al. 2015a). Quantitative real-time PCR was carried out using the QuantStudio 6 Flex System (Applied Biosystems) as described (Cansby et al. 2013) (Supplementary Table 2).

\section{Statistical analysis}

Statistical significance between groups was calculated with an unpaired two-tailed Student's $t$ test or by two-way ANOVA followed by $t$ test for post hoc analysis. A $P<0.05$ was considered statistically significant.

\section{Results}

\section{STK25 coats lipid droplets in 3T3-L1 adipocytes}

Our previous studies have shown that STK25 is associated with intrahepatocellular lipid droplets in mouse and human liver cells (Amrutkar et al. 2015b, 2016b). It is difficult to assess subcellular localization of STK25 in fully differentiated adipocytes in tissue sections. However, we now determined the cellular localization of STK25 
by immunofluorescence analysis in 3T3-L1 adipocytes. STK25 was predominantly present on the surface of lipid droplets, lining both large and small droplets, with the majority of droplets showing positive staining (Fig. 1A). Furthermore, we found that STK25 largely colocalized with lipid droplet-associated protein ATGL (Fig. 1B).

\section{STK25 overexpression in mice aggravates high-fat diet-induced lipid accumulation, inflammatory infiltration and fibrosis in brown and white adipose depots}

The Stk25 transgenic mice used in this study overexpress STK25, to a different extent, in all tissues (Cansby et al. 2013). Our previous investigations revealed that STK25 mRNA and protein abundance is increased by approximately 6-fold in the WAT of high-fat-fed Stk25 transgenic vs WT mice (Cansby et al. 2013). We also found about 10-fold overexpression of STK25 mRNA and protein in the BAT isolated from high-fat-fed Stk25 transgenic mice (Supplementary Fig. 2A and B).

After 18 weeks of high-fat feeding, the interscapular BAT of Stk25 transgenic mice was visibly more lipid laden, displaying a milky appearance, compared with the BAT from WT controls (Fig. 2A). Consistently, histologic examination of H\&E-stained BAT sections revealed the frequent presence of large, unilocular lipid droplets in the brown adipocytes of Stk25 transgenic mice, rather than the classic multilocular fat droplets seen in the majority of the brown adipocytes in WT controls (Fig. 2B). Histomorphometric analysis also confirmed a shift in the overall lipid droplet size distribution toward significantly larger droplets in Stk25 transgenic BAT (Fig. 2C). Inflammation in adipose tissue, characterized primarily by macrophage infiltration, plays a key role in linking obesity with systemic insulin resistance and whole-body metabolic dysfunction (Xu et al. 2003, Lumeng \& Saltiel 2011). Notably, we found aggravated macrophage infiltration, as studied by the staining for F4/80 and Gr1 (Ly6C), in the BAT sections of high-fat-fed Stk25 transgenic vs WT mice (Fig. 2B and C). Many studies have also unveiled a close link between the pathogenesis of chronic inflammation and the development of fibrosis, a hallmark of dysfunctional adipose tissue in the obese state (Sun et al. 2013). Consistent with an increase in inflammation, we observed a $1.6 \pm 0.4$-fold exacerbated fibrosis, as studied by Picrosirius Red staining for collagen fibers, in the BAT sections of highfat-fed Stk25 transgenic mice (Fig. 2B and C). Despite this convincing morphological evidence for the whitening of BAT in Stk25 transgenic mice, the mRNA levels of BAT-specific markers (Ucp1, Prdm16, Ppargc1a (also known as Pgc1 $\alpha$ ), Dio2, Cidea and $\mathrm{Cidec}$ ) were not repressed in Stk25 transgenic BAT compared to WT BAT (Supplementary Fig. 3).

Our previous studies have shown that, upon a chronic exposure to dietary lipids, the size of white adipocytes in the subcutaneous deposits of Stk25 transgenic mice is increased compared with respective to WT controls (Cansby et al. 2013). Consistent with an increased size of white adipocytes, we now also observed an enhanced presence of localized macrophages, identified by staining for F4/80 and Gr1 (Ly6C), in the crown-like structures surrounding adipocytes in the sWAT of high-fat-fed Stk25
A

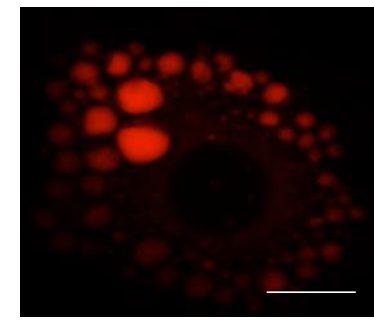

B

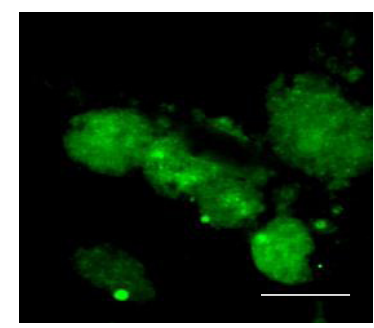

Nile Red

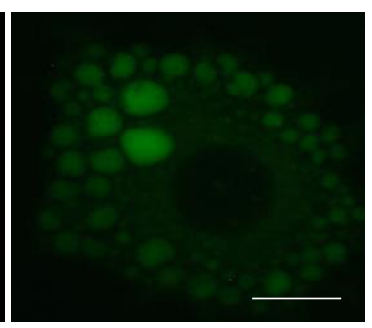

ATGL

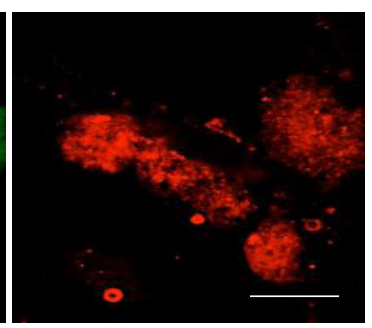

Merge

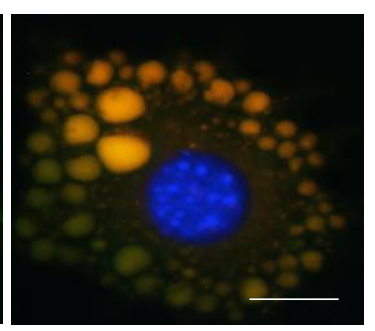

Merge

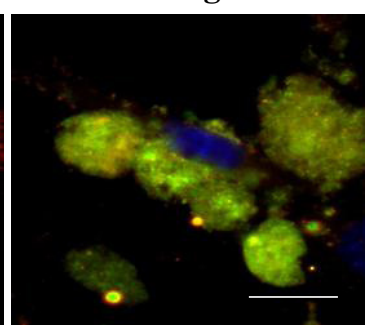

Figure 1

STK25 coats lipid droplets in adipocytes. (A) Representative images of 3T3-L1 adipocytes stained with Nile Red for lipids (green) and processed for immunohistochemistry using anti-STK25 (red). (B) Representative images of 3T3-L1 adipocytes processed for immunohistochemistry using anti-STK25 (green) or anti-ATGL (red) antibodies. Merged image shows colocalization in yellow; nuclei stained with DAPI (blue). Scale bars, $20 \mu \mathrm{m}$. 
A

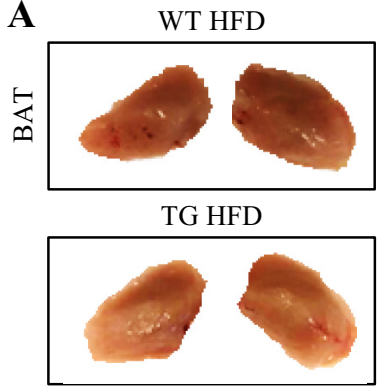

B
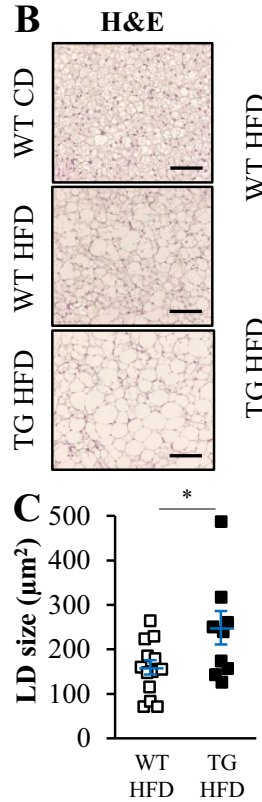
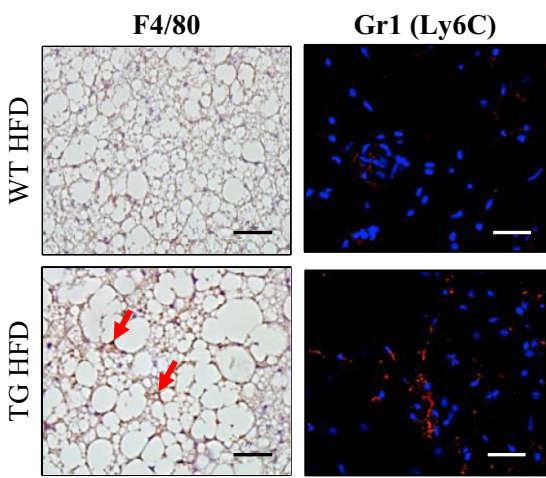

Picrosirius Red
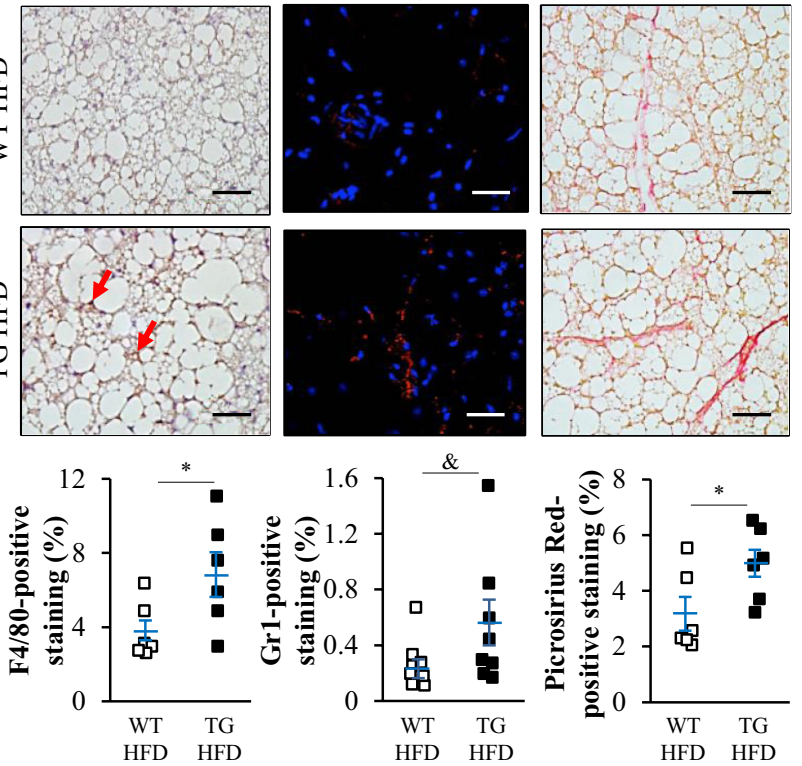
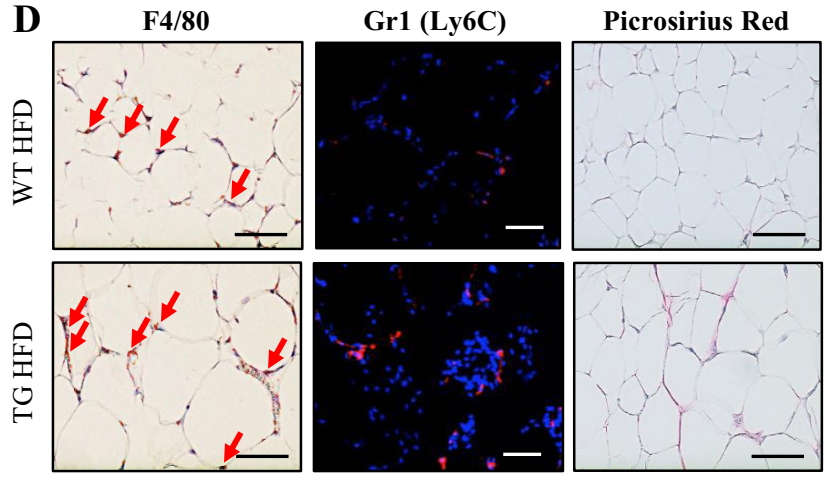

E
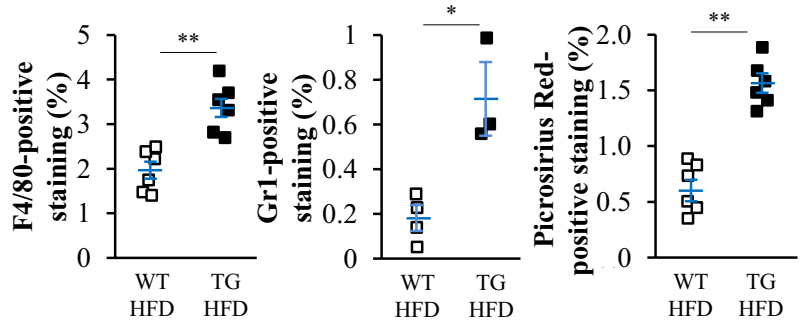

Figure 2

Aggravated lipid accumulation, inflammatory infiltration and fibrosis in adipose tissue of high-fat-fed Stk25 transgenic mice. (A) Representative images of interscapular BAT. (B and D) Representative sections of Stk25 transgenic and WT BAT (B) or SWAT (D) stained with H\&E or Picrosirius Red or processed for immunohistochemistry using anti-F4/80 antibodies or for immunofluorescence using anti-Gr1 (Ly6C) antibodies (pink); nuclei stained with DAPI (blue). In (B), H\&E-stained BAT sections from chow-fed control mice of the same age are shown for comparison. Scale bars, $50 \mu \mathrm{m}$. (C and E) Quantification of lipid droplet size, F4/80, Gr1 (Ly6C) and Picrosirius Red staining in BAT (C) and sWAT (E) sections. Data are mean \pm s.E.M. from 6 to 12 mice per genotype except for quantification of $\mathrm{Gr} 1(\mathrm{Ly} 6 \mathrm{C})$ in sWAT, where $n=3-4$. HFD, high-fat diet; LD, lipid droplet; $\mathrm{TG}$, transgenic. ${ }^{*} P<0.1 ;{ }^{*} P<0.05$; $* * P<0.01$.

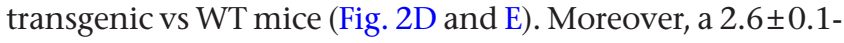
fold aggravated fibrosis, defined by Picrosirius Red staining, was seen in the sWAT of high-fat-fed Stk25 transgenic mice (Fig. 2D and E). Despite these morphological differences, the mRNA levels of WAT-specific markers Tcf21, Hoxc9, Pparg and Fabp4 were similar between the genotypes (Supplementary Fig. 3). Furthermore, we found that the mRNA expression of leptin and adiponectin in the sWAT and eWAT was similar comparing Stk25 transgenic and WT mice (Supplementary Fig. 3). Of note, we have previously reported slightly higher circulating leptin levels in high-fatfed Stk25 transgenic vs WT mice (Nunez-Duran et al. 2017) while fasting plasma adiponectin concentrations were similar between the genotypes (Supplementary Fig. 4A).

\section{Stk25 transgenic mice display impaired mitochondrial function in brown and white adipose tissues}

BAT is characterized by a high mitochondrial content and requires $\beta$-oxidation for fuel heat generation (Harms \& Seale 2013). Our previous findings suggesting a repressed mitochondrial function in the liver and skeletal muscle of high-fat-fed Stk25 transgenic mice (Amrutkar et al. 2015b, 
2016a, Chursa et al. 2017) prompted us to hypothesize that aggravated lipid storage in the transgenic BAT is caused by an impairment of mitochondrial content and/or activity. Indeed, we found that staining with MitoTracker Red, a fluorescent dye that specifically accumulates within respiring mitochondria, was $2.0 \pm 0.1$-fold lower in the histological BAT sections of high-fat-fed Stk25 transgenic vs WT mice (Fig. 3A and B).

To further quantify the mitochondrial activity in relation to lipid deposition in an intact tissue matrix of the BAT, TPEF and CARS microscopy were used. TPEF was employed to quantify active mitochondria in living cells stained with rhodamine 123, while CARS targets molecular vibrations in the alkyl backbone of the TAGs in lipid droplets by two pulsed laser beams over-layed in time and space. CARS microscopy (Zumbusch et al. 1999) has been broadly used to study lipid biology in different cell types (Evans \& Xie 2008, Mouras et al. 2011, Brannmark et al. 2014), whereas an addition of the mitochondrial staining allows to track the dynamics in the relative amount of lipids vs the active mitochondria inside the living cells and to estimate 'the metabolic activity index' as the ratio of mitochondrial activity over lipid content. We found that the mitochondrial activity assessed by TPEF was significantly repressed, and the lipid storage assessed by CARS was increased, in the BAT from high-fat-fed Stk25 transgenic vs WT mice, resulting in a 3.5 \pm 1 .4-fold decrease in the metabolic activity index (Fig. 3C and D).

Consistent with our findings in BAT, the mitochondrial activity was significantly repressed, and the lipid content was increased, as measured by TPEF and CARS, respectively, in both sWAT and eWAT from highfat-fed Stk25 transgenic mice compared with WT controls, resulting in about 2 -fold decrease in the metabolic activity index (Fig. 3E and F). Since reduced recruitment of lipids for oxidation may relate to the decrease in lipolytic activity, we next compared the specific activity of main adipose tissue lipases in the WAT. ATGL initiates the breakdown of lipids in adipose tissue, followed by the action of the major diglyceride lipase - HSL (Rosen \& Spiegelman 2014). Indeed, we found that the activity of ATGL and HSL was repressed by $1.4 \pm 0.1$ - and 2.2 \pm 0.1 -fold, respectively, in the eWAT of high-fat-fed Stk25 transgenic vs WT mice (Fig. $3 \mathrm{G}$ and $\mathrm{H}$ ).

Electron micrographs failed to show any ultrastructural abnormalities in the BAT or WAT mitochondria of Stk25 transgenic mice (Supplementary Figs 5A, B and C and 6). Moreover, we saw no alterations in proteins of the mitochondrial oxidative phosphorylation (OXPHOS) pathway (i.e., core catalytic components of electron transport chain complexes and ATP synthase) or the abundance of acetyl-CoA carboxylase (ACC)/phosphoACC, a key regulator of both mitochondrial lipid oxidation and lipid synthesis, in the BAT or WAT comparing the genotypes (Supplementary Fig. 7A, B, C and D). It has previously been demonstrated that inadequate conclusions may be reached if $U c p 1$ mRNA expression is used as a proxy for estimating the thermogenic capacity of BAT (Nedergaard \& Cannon 2013), underscoring the importance of confirming any changes in mRNA levels with the measurements of UCP1 protein abundance. Although Ucp1 mRNA was slightly increased in the BAT from Stk25 transgenic mice (Supplementary Fig. 3), we found no alterations in the levels of proteins, which regulate the thermogenic action of BAT - UCP1, type 2 iodothyronine deiodinase (DIO2), or the fatty acid elongase ELOVL6 - when comparing the BAT from Stk25 transgenic vs WT mice (Supplementary Fig. 7B).

Toxic lipid intermediates such as ceramides as well as endoplasmic reticulum (ER) and oxidative stress have emerged as key factors, which can trigger mitochondrial dysfunction and inflammation in different tissues (Zhang \& Kaufman 2008, Wang \& Kaufman 2016, Sunny et al. 2017). Here, we found that the tissue concentrations of ceramides or dihydroceramides were not altered in the BAT or eWAT from Stk25 transgenic mice compared with WT controls (Supplementary Fig. 8A). The mRNA levels of the major indicators of ER stress response Chop (also known as Ddit3), Bip (also known as Hspa5 or Grp78) and Edem1 were also largely similar comparing the genotypes, although Chop and Edem1 were slightly elevated in Stk25 transgenic BAT and sWAT, respectively (Supplementary Fig. 3). Interestingly, we found 1.6 \pm 0.2 -fold higher concentrations of TBARS, a classic marker of oxidative stress, in the eWAT from Stk25 transgenic mice (Supplementary Fig. 9A).

STK25 depletion in mice protects against high-fat diet-induced lipid accumulation, meta-inflammation and fibrosis in brown and white adipose depots

The Stk25-/- mice used in this study are depleted of STK25 protein in all organs including BAT and WAT (Matsuki et al. 2013, Amrutkar et al. 2015a). Upon dissection after 18 weeks of high-fat-feeding, the interscapular BAT of Stk25-l- mice displayed the dark brown color characteristic to the BAT of chow-fed mice, in contrast to the visibly whitish BAT from high-fat-fed WT littermates (Fig. 4A). Consistently, histologic analysis showed that the BAT from high-fat-fed Stk25-1- mice was composed of 


\begin{tabular}{|l|l|l|l|l|}
$\begin{array}{l}\text { Journal of } \\
\text { Endocrinology }\end{array}$ & S sütt, E Cansby et al. & $\begin{array}{l}\text { STK25 in adipose tissue } \\
\text { dysfunction in obesity }\end{array}$ & $\mathbf{2 3 8 : 3}$ & $\mathbf{1 9 3}$ \\
\hline
\end{tabular}
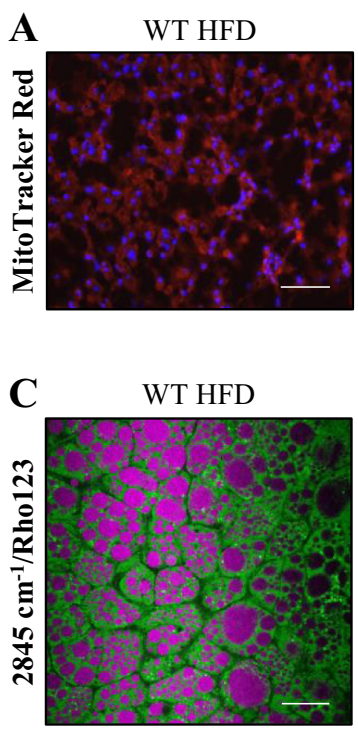

D.

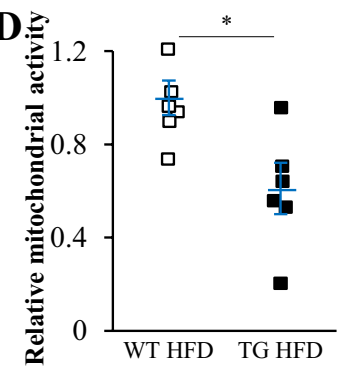

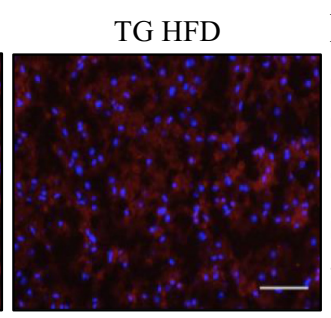

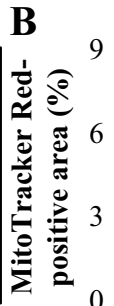

TG HFD
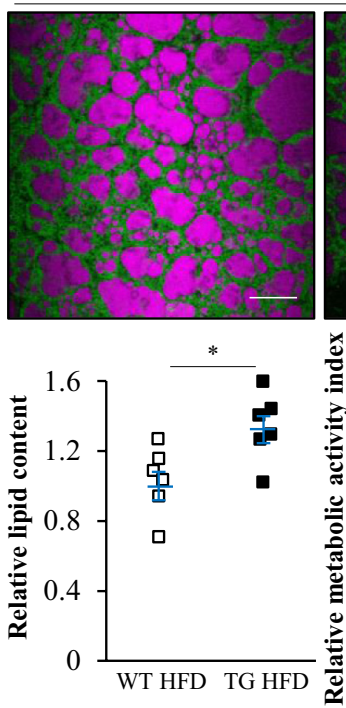

$\mathbf{E}$
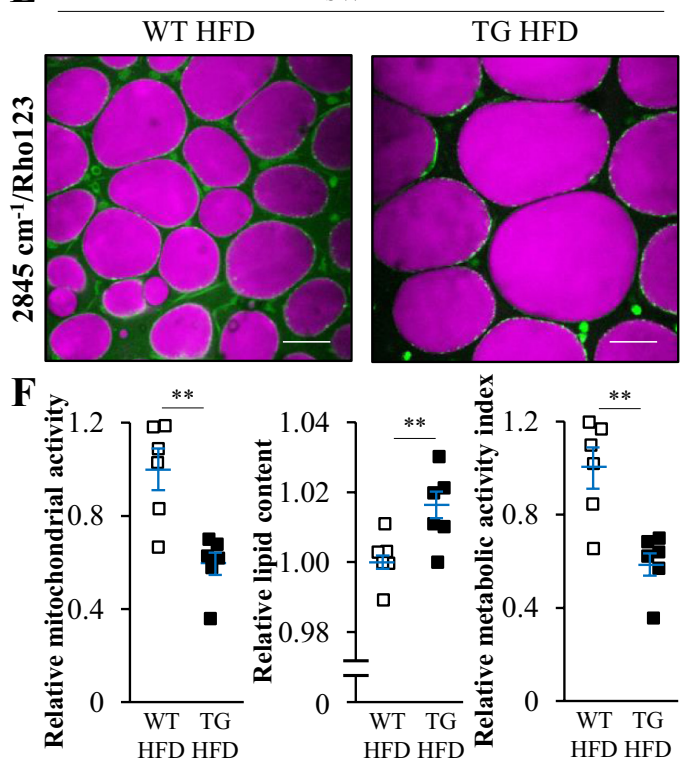
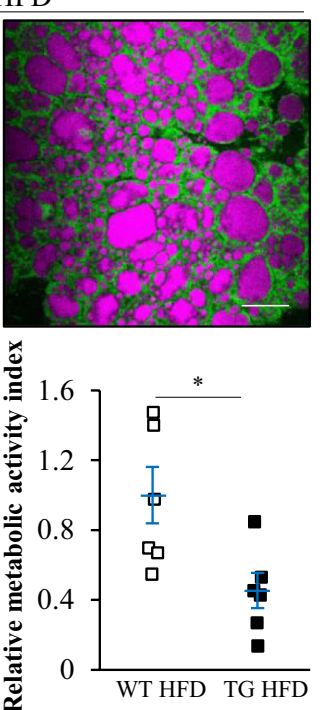

G

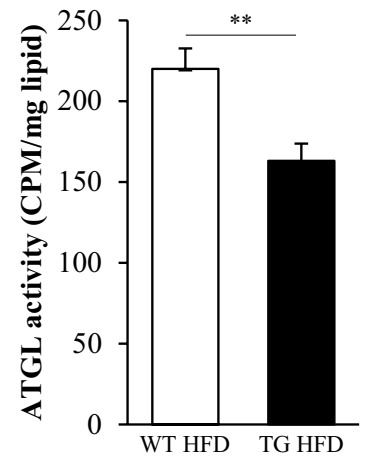

H

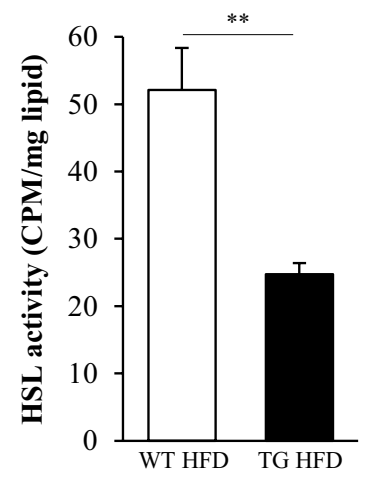

Figure 3

Impaired mitochondrial function in adipose tissue of high-fat-fed Stk25 transgenic mice. (A) Representative sections of Stk25 transgenic and WT BAT stained with MitoTracker Red; nuclei stained with DAPI (blue). Scale bars, $50 \mu \mathrm{m}$. (B) Quantification of MitoTracker Red staining in BAT sections. (C and E) Representative images showing lipids (CARS, 2845/cm; pink) and active mitochondria (TPEF, Rho123; green) in living BAT (C) and sWAT/eWAT (E). Scale bars, $50 \mu \mathrm{m}$. (D and F) Quantification of cellular mitochondrial activity and lipid content in BAT (D) and sWAT/eWAT (F). The metabolic activity index is estimated as the ratio of mitochondrial activity over lipid content with a higher index indicating a metabolically more active adipose tissue. (G and $\mathrm{H}$ ) ATGL (G) and HSL activity (H) in adipocytes isolated from eWAT. Data are mean \pm S.E.M. from 6 to 8 mice per genotype. CPM, counts per minute; HFD, high-fat diet; Rho123, rhodamine 123; TG, transgenic. ${ }^{*} P<0.05 ; * P<0.01$ 

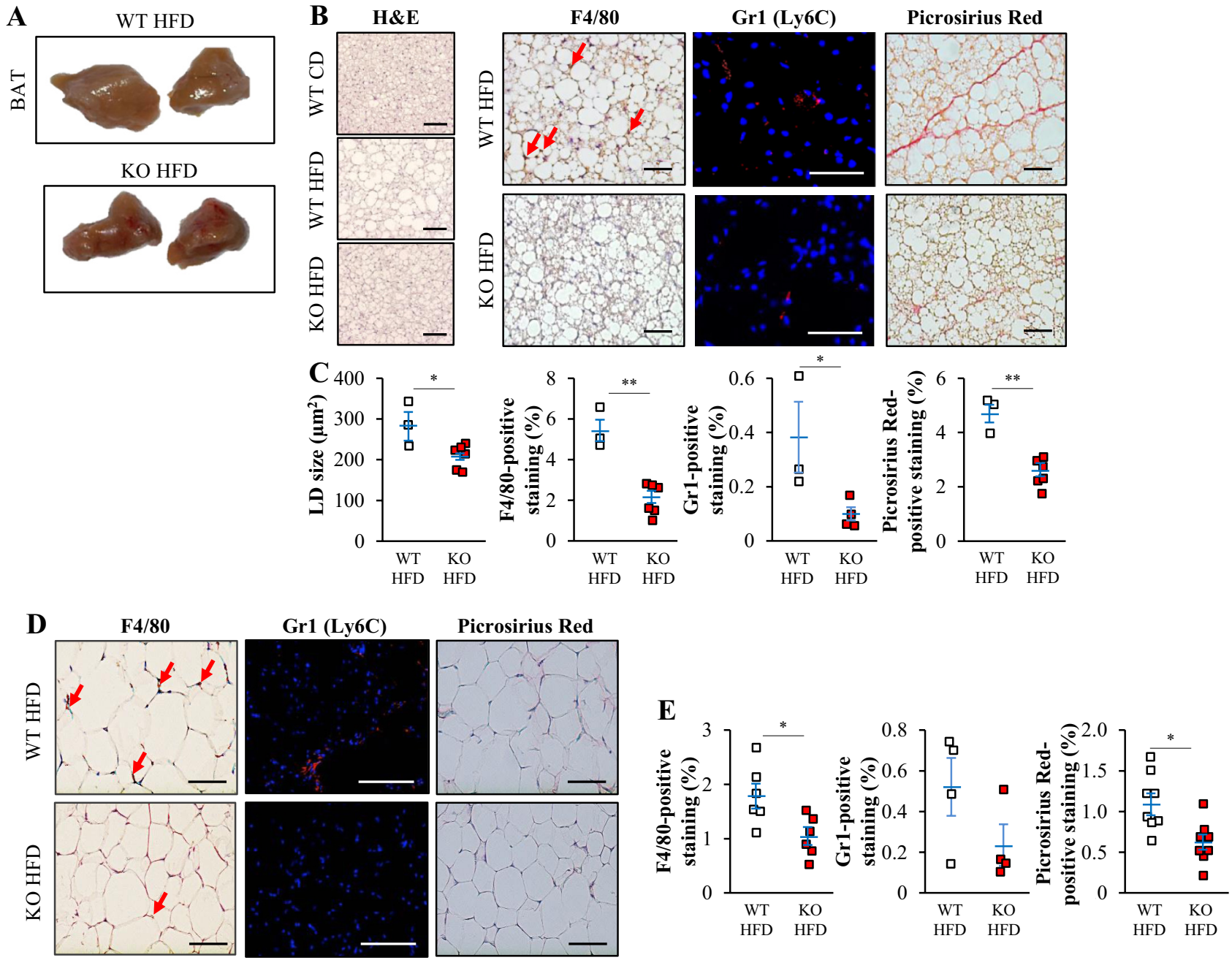

\section{Figure 4}

Reduced lipid accumulation, inflammatory infiltration, and fibrosis in adipose tissue of high-fat-fed Stk25-- mice. (A) Representative images of interscapular BAT. (B and D) Representative sections of Stk25 knockout and WT BAT (B) or SWAT (D) stained with H\&E or Picrosirius Red or processed for immunohistochemistry using anti-F4/80 antibodies or for immunofluorescence using anti-Gr1 (Ly6C) antibodies (pink); nuclei stained with DAPI (blue). In (B), H\&E-stained BAT sections from chow-fed control mice of the same age are shown for comparison. Scale bars, $50 \mu \mathrm{m}$. (C and E) Quantification of lipid droplet size, F4/80, Gr1 (Ly6C) and Picrosirius Red staining in BAT (C) and sWAT (E) sections. Data are mean \pm S.E.M. from 3 to 8 mice per genotype. HFD, high-fat diet; KO, knockout; LD, lipid droplet. ${ }^{*} P<0.05 ;{ }^{* *} P<0.01$

small polygonal mitochondria-enriched adipocytes with numerous very small lipid droplets, which was similar to the chow-fed controls but in marked contrast to enlarged lipid droplets observed in the brown adipocytes of highfat-fed WT mice (Fig. 4B and C). Notably, Stk25-/- mice were protected from high-fat diet-induced macrophage infiltration and fibrosis in the BAT (Fig. 4B and C). Despite these marked differences in morphology, the mRNA levels of BAT-specific markers (Ucp1, Prdm16, Pgc1 $\alpha$, Dio2, Cidea and Cidec) were similar in the Stk25-/- and WT BAT (Supplementary Fig. 10).

Our previous studies have demonstrated a reduced size of adipocytes in the sWAT of high-fat-fed $S t k 25^{-/-}$vs
WT mice (Amrutkar et al. 2015a). We now also observed that Stk25-I- mice were protected against diet-induced macrophage infiltration and fibrosis in the sWAT (Fig. 4D and E). The mRNA levels of WAT-specific markers (Tcf21, Hoxc9, Pparg and Fabp4) were similar between the genotypes (Supplementary Fig. 10). Notably, we found that the mRNA expression of leptin was increased in the WAT from Stk25-I- mice compared with WT littermates without any difference in adiponectin mRNA levels (Supplementary Fig. 10). However, fasting plasma concentrations of both leptin and adiponectin were similar between the genotypes (Supplementary Fig. 4B and C). 


\section{Stk25-/- mice display enhanced mitochondrial}

\section{function in brown and white adipose tissues}

Reciprocally to our results in Stk25 transgenic mice, we observed that the mitochondrial activity assessed by MitoTracker Red staining was $1.4 \pm 0.1$-fold higher in the BAT sections of high-fat-fed Stk25-/- mice compared with WT mice (Fig. 5A and B). Furthermore, mitochondrial DNA (mtDNA) copy number in the BAT extracts was also increased in Stk25-/- mice (Supplementary Fig. 11). Consistent with these findings, combined analysis by TPEF and CARS showed that the mitochondrial activity was significantly enhanced, and reciprocally, the lipid content was markedly reduced, in the BAT from high-fatfed Stk25/- mice compared with WT mice, resulting in a 2.9 \pm 0.2 -fold increase in the metabolic activity index (Fig. 5C and D). Furthermore, we also found that the mitochondrial activity was significantly increased in the sWAT and eWAT from high-fat-fed Stk25-/- mice, resulting in a about 2-fold higher metabolic activity index (Fig. 5E and F). The ATGL and HSL activity was slightly elevated in the eWAT comparing high-fat-fed Stk25-/- vs WT mice (Fig. $5 \mathrm{G}$ and $\mathrm{H}$ ).

Electron micrographs failed to show any ultrastructural abnormalities in the BAT or WAT mitochondria in Stk25-/mice (Supplementary Figs 12A, B and C and 13). Moreover, we found no significant differences in abundance of OXPHOS or ACC proteins in the BAT or WAT comparing the genotypes (Supplementary Fig. 14A, B, C and D). The levels of UCP1, DIO2 and ELOVL6 proteins were also similar in the BAT from Stk25-knockout and WT mice (Supplementary Fig. 14B).

Notably, we found that the tissue concentrations of ceramides were $1.7 \pm 0.2$-fold lower in the eWAT from Stk25-1- vs WT mice (Supplementary Fig. 8B), whereas the levels of oxidative stress marker TBARS were not changed comparing the genotypes (Supplementary Fig. 9B). Interestingly, all the major indicators of ER stress measured (Chop, Bip and Edem1) were about 2-fold lower in the sWAT from Stk25-knockout mice compared with WT controls (Supplementary Fig. 10).

Knockdown of STK25 represses lipid deposition and enhances mitochondrial function in cultured adipocytes

To study the cell-specific role of STK25 in adipocytes and to further elucidate the mode of action of this protein in adipose tissue metabolism, we transfected mouse brown fat cell line HIB-1B with Stk25-specific siRNA or with a nontargeting control (NTC) siRNA. In all experiments, the protein level of STK25 in cells transfected with Stk25 siRNA was below the detection limit of Western blot (Fig. 6A).

We found that lipid accumulation assessed by Oil Red O staining was 1.9 \pm 0.2 -fold lower in HIB-1B cells transfected with Stk25 siRNA (Fig. 6B). siRNA-mediated silencing of STK25 also resulted in marked increase in $\beta$-oxidation (Fig. 6C) and consistently, mitochondrial area assessed by the staining with MitoTracker Red was augmented in STK25-deficient cells (Fig. 6D). Notably, no change in fatty acid influx or incorporation of mediaderived $\left[{ }^{14} \mathrm{C}\right]$-labeled oleic acid into intracellular TAG was observed in STK25-deficient HIB-1B cells (Fig. 6E and F).

We have previously demonstrated that STK25 regulates lipid catabolism in mouse and human hepatocytes by controlling release of NEFA from lipid droplets via regulation of lipolytic activity (Amrutkar et al. 2015b, 2016b). Consistent with these results, we found that lipolytic activity was $1.3 \pm 0.1$-fold higher in HIB-1B cells transfected with Stk25 siRNA (Fig. 6G). To investigate whether the effect of STK25 depletion on cellular lipid accumulation is mediated solely via regulation of lipolysis, we treated HIB-1B cells with the atheroprotective drug nicotinic acid (NiAc, also called niacin); NiAc blocks intracellular TAG lipolysis and free fatty acid flux in adipose tissue through the G-proteincoupled receptor GPR109A (Tunaru et al. 2003). As expected, NiAc markedly increased lipid storage in HIB-1B cells (Supplementary Fig. 15). Interestingly, we found that lipid accumulation (as assessed by Oil Red O staining) was reduced to a similar extent in cells transfected with Stk25 siRNA in the absence or presence of NiAc (by $14.7 \pm 3.0 \%$ and $20.1 \pm 4.4 \%$, respectively, compared with cells transfected with NTC siRNA; Fig. $6 \mathrm{H}$ ). These results suggest that increased lipolysis is not the only factor that contributes to reduced lipid accumulation in conditions where STK25 is depleted.

Importantly, we found that the mRNA expression of key factors related to differentiation of HIB-1B -

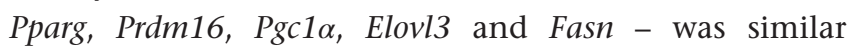
in cells transfected with Stk25 siRNA compared with cells transfected with NTC siRNA, and the mRNA level of Fabp4 was slightly higher, which suggests that the process of differentiation was not impaired in STK25-deficient HIB-1B cells (Supplementary Fig. 16). We found no alterations in abundance of ACC/phospho-ACC, AMP-activated protein kinase (AMPK)/phospho-AMPK, UCP1 or OXPHOS proteins in HIB-1B cells transfected with Stk25 siRNA compared with cells transfected with NTC siRNA (Supplementary Fig. 17A and B). 


\begin{tabular}{l|l|l|c|c|}
\hline $\begin{array}{l}\text { Journal of } \\
\text { Endocrinology }\end{array}$ & S Sütt, E Cansby et al. & $\begin{array}{l}\text { STK25 in adipose tissue } \\
\text { dysfunction in obesity }\end{array}$ & $\mathbf{2 3 8 : 3}$ & $\mathbf{1 9 6}$ \\
\hline
\end{tabular}
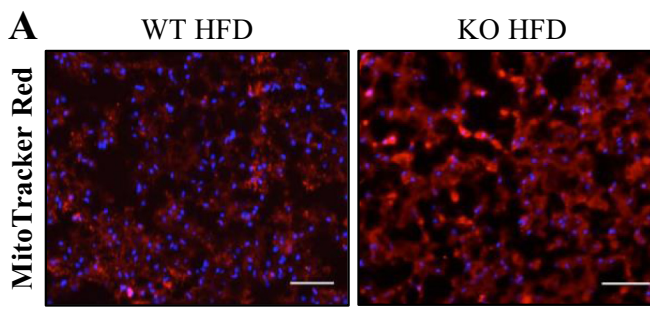

B

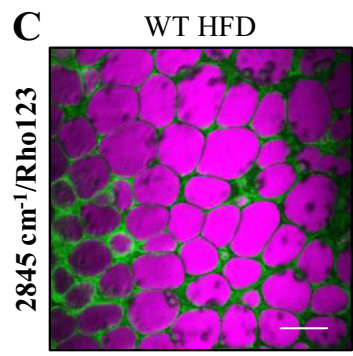

D.:

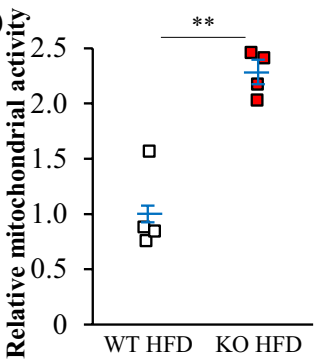

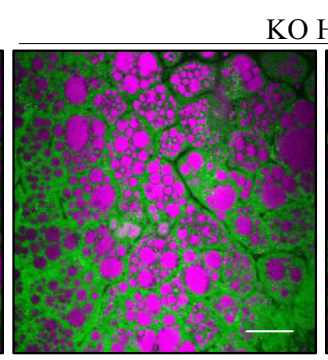

KO HFD

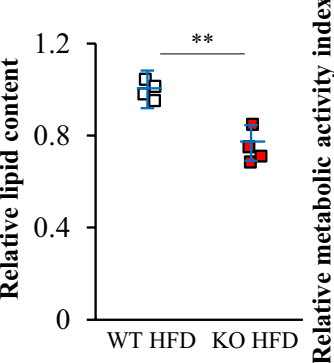

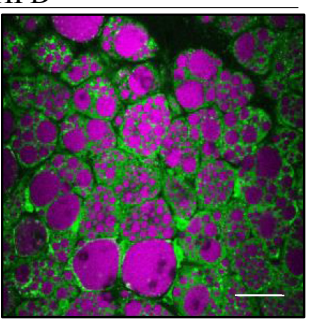
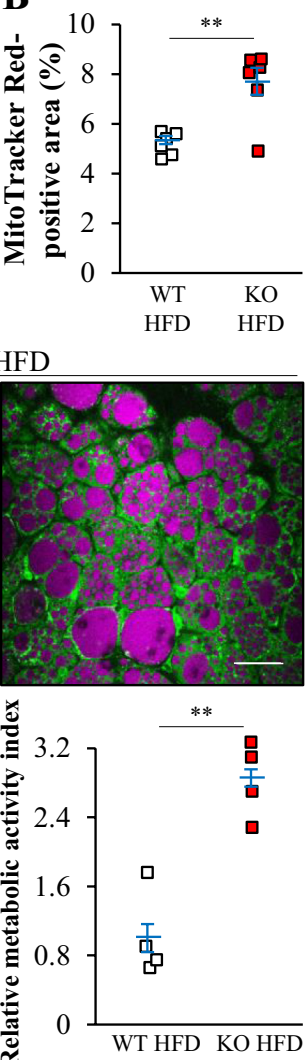

G

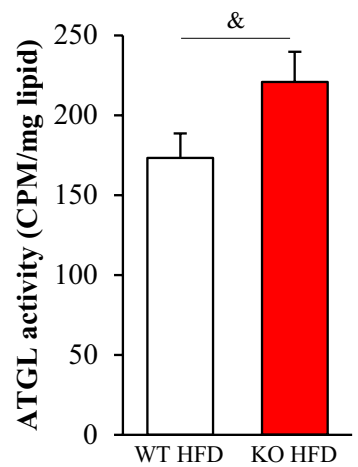

H

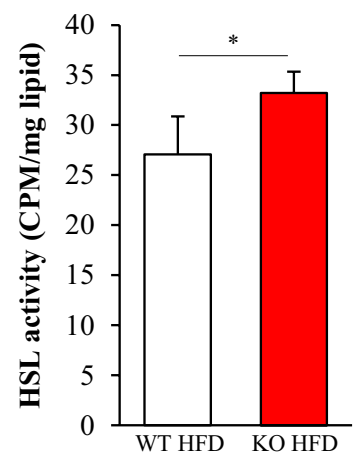

E

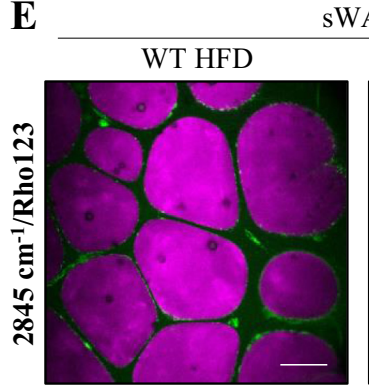

F

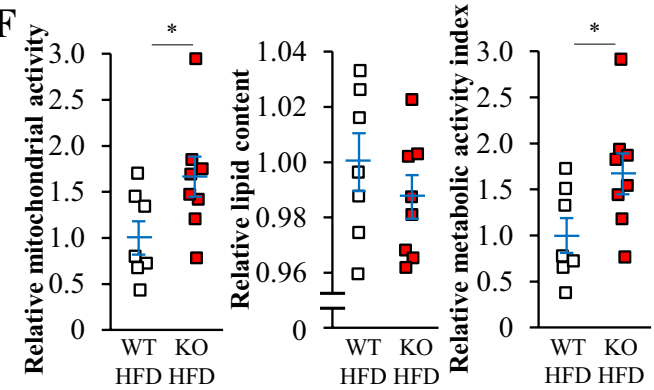

KO HFD

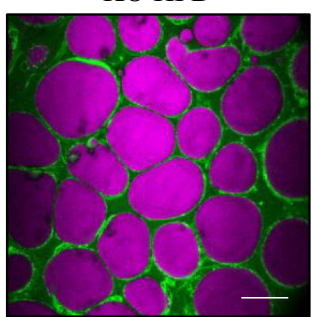

HFD HFD

HFD HFD
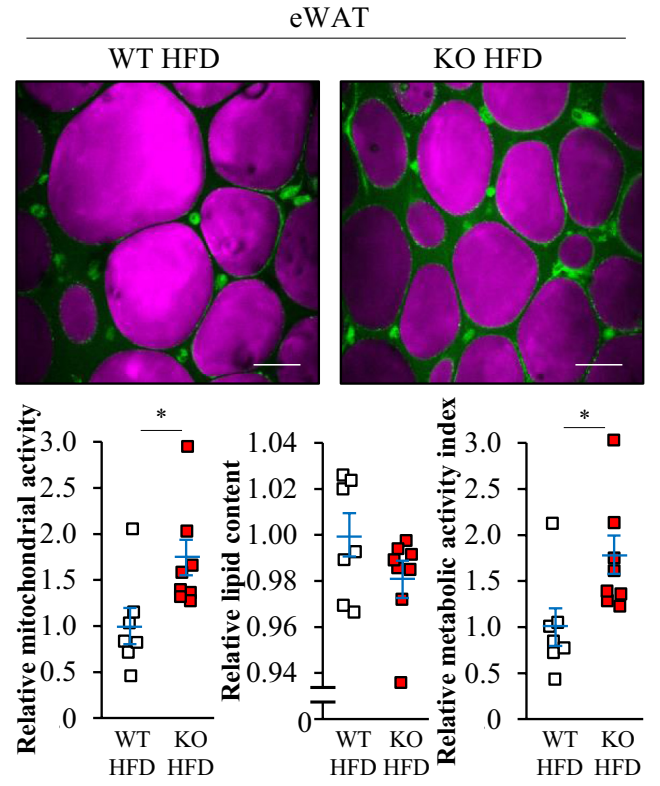

Figure 5

Enhanced mitochondrial function in adipose tissue of high-fat-fed Stk25-l- mice. (A) Representative sections of Stk25-knockout and WT BAT stained with MitoTracker Red; nuclei stained with DAPI (blue). Scale bars, $50 \mu \mathrm{m}$. (B) Quantification of MitoTracker Red staining in BAT sections. (C and E)

Representative images showing lipids (CARS, 2845/cm; pink) and active mitochondria (TPEF, Rho123; green) in living BAT (C) and sWAT/eWAT (E). Scale bars, $50 \mu \mathrm{m}$. (D and F) Quantification of cellular mitochondrial activity and lipid content in BAT (D) and sWAT/eWAT (F). The metabolic activity index is estimated as the ratio of mitochondrial activity over lipid content with a higher index indicating a metabolically more active adipose tissue. ( $G$ and $H$ ) ATGL (G) and HSL activity (H) in adipocytes isolated from eWAT. Data are mean \pm S.E.M. from 4 to 8 mice per genotype. CPM, counts per minute; HFD, high-fat diet; KO, knockout; LD, lipid droplet; Rho123, rhodamine 123 . \& $P<0.1 ;{ }^{*} P<0.05 ;{ }^{*} P<0.01$ 


\begin{tabular}{l|l|l|c|}
$\begin{array}{l}\text { Journal of } \\
\text { Endocrinology }\end{array}$ & S Sütt, E Cansby et al. & $\begin{array}{l}\text { STK25 in adipose tissue } \\
\text { dysfunction in obesity }\end{array}$ & $\mathbf{2 3 8 : 3}$
\end{tabular}

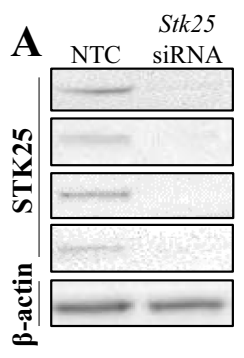

C

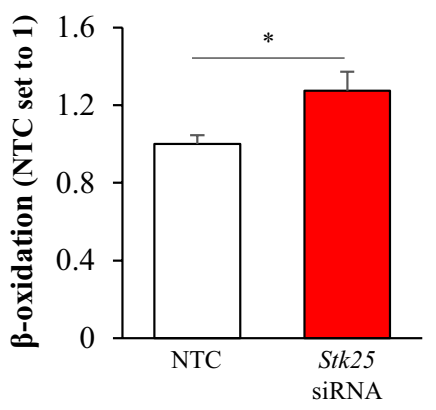

E

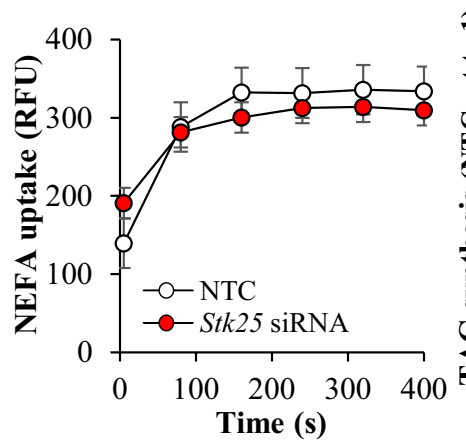

H

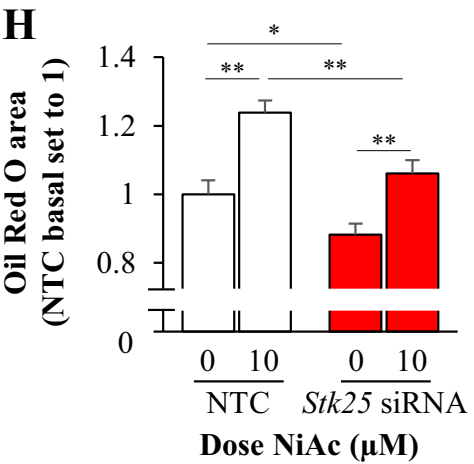

Stk25 siRNA

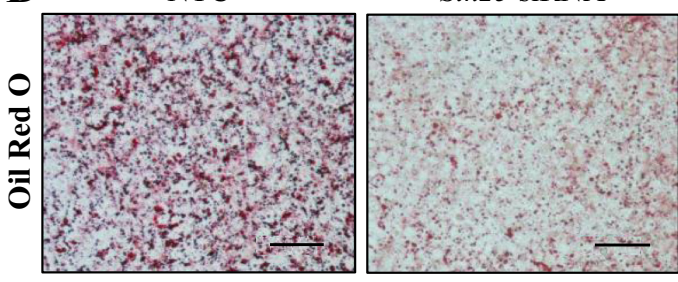

D

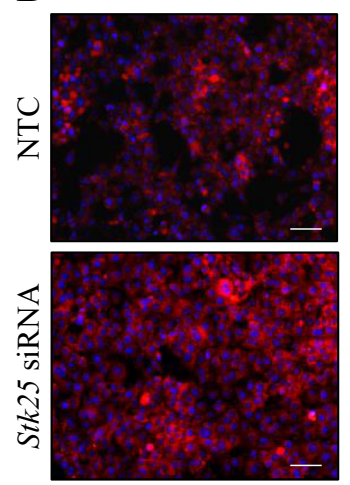

F

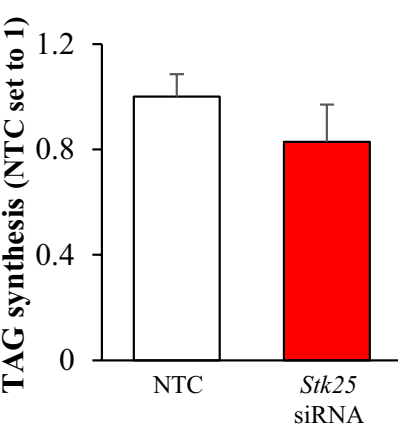

G

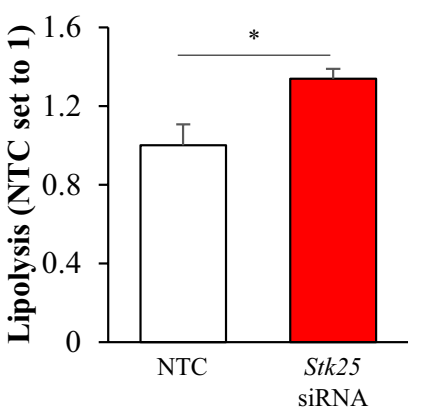

I

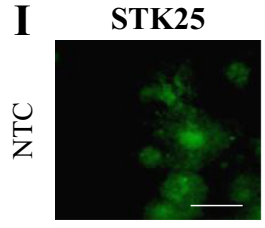

Nile Red

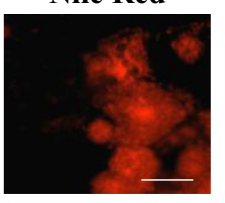

Merge

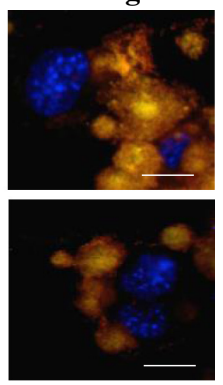

\section{Figure 6}

Suppressed lipid deposition and enhanced mitochondrial function in STK25-deficient cultured adipocytes. (A, B, C, D, E, F, G and H) HIB-1B adipocytes were transfected with Stk25-specific siRNA or with NTC siRNA. (A) Representative Western blot with anti-STK25 antibodies; $\beta$-actin was used as a loading control. (B) Representative cell images stained with Oil Red O for lipids. Scale bars, $100 \mu$ m. Quantification of Oil Red O staining. (C) Oxidation of radiolabeled palmitate. (D) Representative cell images stained with MitoTracker Red. Scale bars, $50 \mu$ m. Quantification of MitoTracker Red staining. (E) Uptake of NEFAs. (F) TAG synthesis from $\left[{ }^{14} \mathrm{C}\right]$-labeled oleic acid. (G) TAG hydrolase activity. (H) Quantification of Oil Red O staining after incubation with $10 \mu \mathrm{M}$ NiAc. (I) 3T3-L1 adipocytes were transfected with Stk25-specific siRNA or with NTC siRNA. Representative cell images stained with Nile Red for lipids (red) or processed for immunohistochemistry using anti-STK25 (green) antibodies. Merged image shows colocalization in yellow; nuclei stained with DAPI (blue). Scale bars, $20 \mu \mathrm{m}$. RFU, relative fluorescence units. ${ }^{\star} P<0.05 ;{ }^{*} P<0.01$. 
Consistent with our findings in HIB-1B cells, we also observed that siRNA-mediated silencing of STK25 resulted in a reduced size of lipid droplets in 3T3-L1 adipocytes (Fig. 6I).

\section{Discussion}

Adipose tissue is the key tissue affected by over-nutrition, and whole-body energy homeostasis critically depends on how well adipose depots remodel in response to an excess of calories. In this study, we describe the novel function of key metabolic mediator - protein kinase STK25 - in metabolic and inflammatory profiling in the context of obesity in two functionally distinct adipose lineages: white adipocytes, which function mainly to store fat, and multilocular brown adipocytes, which function to burn fat for nonshivering thermogenesis. Our previous investigations demonstrate that STK25 regulates the susceptibility to ectopic lipid storage, meta-inflammation and fibrosis in conditions of excess dietary fuels in liver, skeletal muscle and pancreas, controlling the whole-body glucose and insulin homeostasis (Nerstedt et al. 2012, Cansby et al. 2013, Amrutkar et al. 2015a,b, 2016a,b,
Chursa et al. 2017, Nunez-Duran et al. 2017, 2018). Here, we show that STK25 is also a critical determinant of mitochondrial activity, hypertrophy, local inflammatory infiltration and fibrotic damage in the BAT and WAT of obese mice (Fig. 7).

First, we observed a marked morphological difference in the BAT of obese mice with increased or reduced activity of STK25. The adipocytes in Stk25 transgenic BAT accumulated excessive amounts of lipids and lost the typical multilocular morphology, resembling those in WAT with cytoplasm occupied by a large lipid droplet, pushing the nucleus to the cell periphery. Reciprocally, the BAT in Stk25-/- mice was protected from excessive high-fat diet-induced lipid accumulation and resembled the BAT in chow-fed mice. Notably, the mRNA and protein levels of BAT-specific marker genes were similar in the brown adipocytes of all the genotypes, suggesting that these morphological differences were not the result of conversion via change in a transcriptional program. These results in BAT are consistent with our previous observations of an increased or reduced size of white adipocytes in high-fat-fed Stk25 transgenic and knockout mice, respectively (Cansby et al. 2013, Amrutkar et al. 2015a).

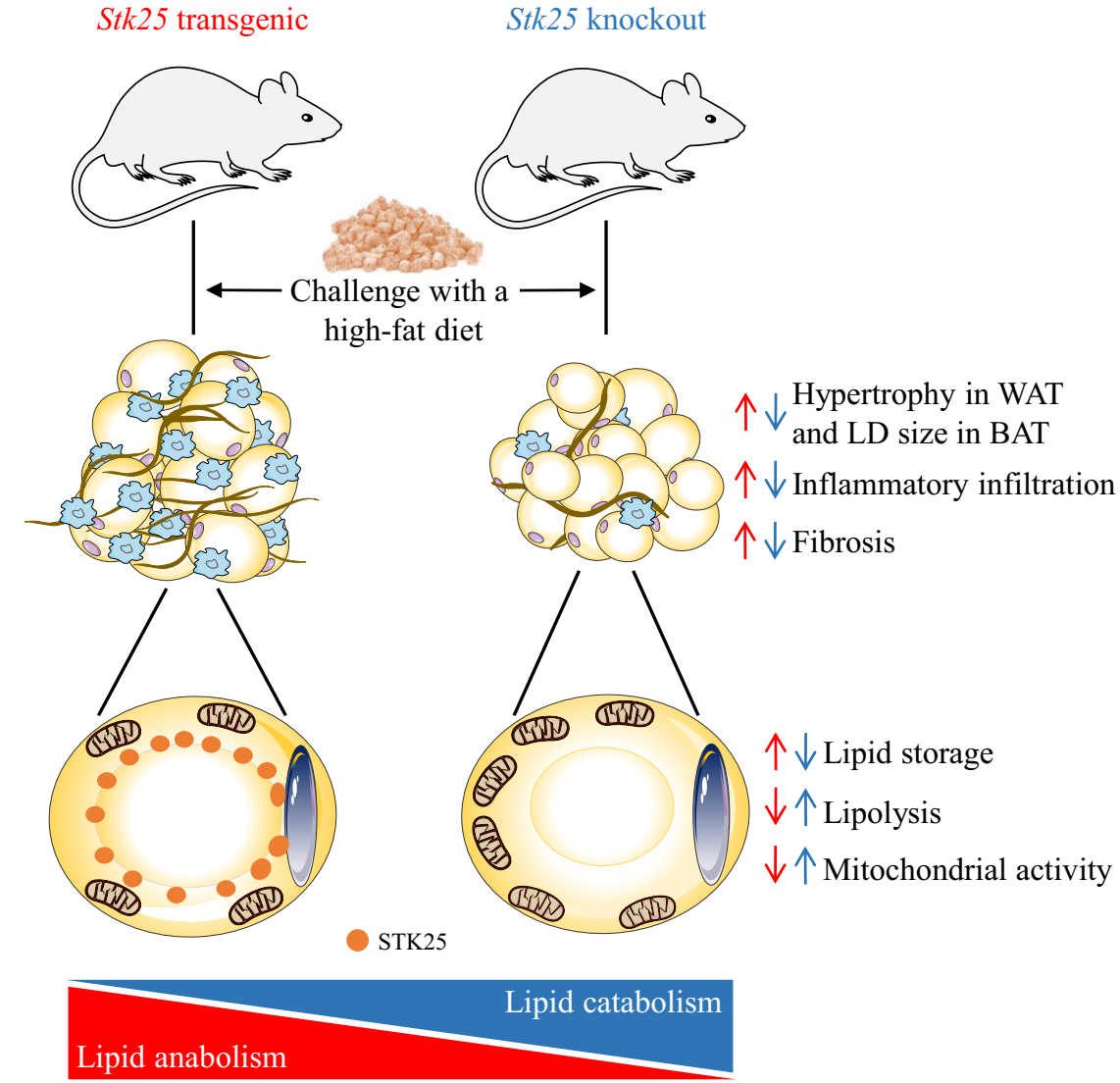

Figure 7

STK25 controls a shift in the metabolic balance of lipid oxidation vs lipid storage in adipose tissue in conditions of excess dietary fuels. Schematic illustration of reciprocal metabolic responses in adipose tissue in transgenic mice overexpressing STK25 (indicated by red arrows) and Stk25-I- mice (indicated by blue arrows) compared with their corresponding WT littermates. Brown lines illustrate collagen deposition; light blue cells illustrate adipose tissue macrophages. LD, lipid droplets. 
One possible explanation for these reciprocal differences in lipid accumulation observed in the adipocytes of Stk25 transgenic vs knockout mice is a change in adipose tissue energy expenditure via altered mitochondrial activity. Indeed, we found that highfat-fed Stk25 transgenic and knockout mice displayed markedly repressed or enhanced mitochondrial function, respectively, in both BAT and WAT depots, compared with respective WT controls. Notably, there is growing evidence suggesting that an impaired mitochondrial function is a causative factor in adipose tissue dysfunction in obesity, contributing to the progression of type 2 diabetes. Recent clinical investigations show that in humans, the WAT from obese individuals displays a downregulation in mitochondrial activity and fatty acid oxidation, with the latter inversely correlating with insulin resistance (Heinonen et al. 2015). Consistently, loss of mtDNA, reduction in the number of mitochondria and impaired mitochondrial respiration have been reported in white adipocytes from $\mathrm{db} / \mathrm{db}$ mice (Choo et al. 2006). Furthermore, a WAT-specific depletion of Tfam (the gene encoding mitochondrial transcription factor A) in mice increases mitochondrial fatty acid oxidation, which protects mice from diet-induced insulin resistance (Vernochet et al. 2012). Even though the BAT depot is present in relatively small amounts compared with WAT, it has a high mitochondrial content and capacity for $\beta$-oxidation, and consequently, the activated BAT is considered to significantly contribute to the whole-body metabolism (Nedergaard et al. 2010). Indeed, in a mouse model of 'human-like' lipoprotein metabolism, metformin was shown to increase BAT mitochondrial content and activity, implicating BAT in the actions of this widely used anti-diabetic drug (Geerling et al. 2014). In light of these previous studies, our results suggest that pharmacological inhibition of STK25 activity would potentially provide a strategy to protect BAT and WAT against diet-induced dysfunction via enhanced mitochondrial function, with beneficial effects on whole-body metabolic homeostasis.

Preventing or reversing local adipose tissue inflammation is one of the therapeutic strategies, which is attempted to counteract diet-induced metabolic dysfunction. A potent negative impact of adipose tissuederived inflammation on systemic glucose homeostasis is highlighted by recent data that demonstrate that selectively silencing inflammatory genes in WAT macrophages leads to a significant improvement in wholebody glucose tolerance (Aouadi et al. 2013). Furthermore, many studies have unveiled close links between the pathogenesis of chronic inflammation in adipose tissue and the development of fibrosis due to abnormal collagen deposition (Sun et al. 2013). Notably, in obese human adipose tissue, large areas of fibrosis can be found along with infiltration of adipose tissue macrophages (Pasarica et al. 2009, Spencer et al. 2010). In this study, we found that high-fat-fed Stk25 transgenic and knockout mice displayed markedly aggravated or repressed macrophage infiltration and fibrosis, respectively, both in BAT and WAT depots, which likely contributed to the previously reported impaired vs improved systemic glucose and insulin homeostasis in these mice (Cansby et al. 2013, Amrutkar et al. 2015a).

The global overexpression or depletion of STK25 in transgenic and knockout mice, respectively, does not allow us to address whether the impact of STK25 on lipid homeostasis and mitochondrial function in the BAT and WAT is direct or secondary to the action of STK25 in tissues other than adipose tissue. However, in this study, we also found that repression of excessive lipid accumulation seen in Stk25-l- BAT and WAT was replicated in HIB-1B and 3T3-L1 adipocytes, where STK25 was depleted by siRNA, which demonstrates an autonomous action of STK25 in adipocytes. Furthermore, we characterized the mode of action of STK25 in the regulation of lipid partitioning in adipose tissue by showing that STK25 depletion in HIB-1B cells increased lipid catabolism (lipolysis and $\beta$-oxidation) without any alterations in lipid anabolism (NEFA uptake and TAG synthesis). Notably, in spite of our results showing increased lipolysis in HIB-1B adipocytes transfected with Stk25 siRNA as well as in adipocytes isolated from Stk25-knockout WAT, the high-fat-fed Stk25-/- mice displayed lower rather than higher levels of circulating free fatty acids compared with WT littermates (Supplementary Fig. 18), which likely relates to a simultaneous increase in oxidative capacity in the skeletal muscle, liver as well as adipose tissue of Stk25-/- mice (this study; (Amrutkar et al. $2015 a, 2016 b)$ ).

We have previously reported that ACC protein abundance is significantly reduced in the livers of highfat-fed mice where STK25 activity is repressed by genetic depletion or ASO treatment, whereas ACC levels are higher in the livers of high-fat-fed Stk25 transgenic mice compared with WT controls (Cansby et al. 2013, Amrutkar et al. 2015a, Nunez-Duran et al. 2018). This provides a likely mechanism for STK25 to regulate liver lipid retention and mitochondrial activity since the enzymatic product of ACC, malonyl-CoA, is a precursor of fatty acid synthesis and also represses long-chain fatty acyl-CoA oxidation in mitochondria through allosteric inhibition of carnitine palmitoyltransferase (CPT) (Savage et al. 2006). 
However, in this study we did not detect any alterations in protein abundance of ACC in the BAT or WAT from Stk25 transgenic or knockout mice compared with WT controls or in HIB-1B cells where STK25 was depleted, suggesting that changes of ACC levels are not involved in regulation of lipid metabolism by STK25 in adipose tissue. Interestingly, we found lower levels of ceramides and indicators of ER stress in Stk25-knockout WAT, and higher levels of oxidative stress marker TBARS in Stk25 transgenic WAT, compared with their corresponding WT controls. While these results indicate that alterations in lipotoxicity, ER and oxidative stress may have contributed to the observed phenotype, further studies are needed to fully characterize the molecular mechanisms by which STK25 regulates mitochondrial activity and lipid partitioning in adipose tissue.

Our previous studies have shown that challenge with a high-fat diet does not change the endogenous levels of STK25 protein in mice in major metabolic tissues: skeletal muscle, liver, and WAT, although the Stk25 mRNA expression was slightly increased in the WAT of high-fatfed mice compared with chow-fed counterparts (Cansby et al. 2013). Here, we assessed STK25 expression in mice in response to fasting/re-feeding and found that STK25 mRNA and protein levels were not significantly different in the BAT or WAT samples collected after 12-hour fasting compared with re-feeding (Supplementary Fig. 19A and B). We also examined the expression of human STK25 mRNA in relation to obesity in a previously published microarray experiment performed in WAT obtained from 26 non-obese and 30 obese women (Arner et al. 2012); however, we did not detect any differences comparing these two groups (Supplementary Fig. 20). Thus, the physiological regulation of STK25 in adipose tissue in vivo remains elusive.

There is a huge unmet medical need for effective pharmacological interventions that are capable of preventing and/or reversing the whole-body diabetic phenotype; this is likely to involve discoveries of new targets rather than modifications of the established drug candidates, for which further improvements are considered to be only marginal (Kusminski et al. 2016). In addition to our previous findings showing that STK25 regulates the susceptibility to diet-induced ectopic lipid storage and insulin resistance in liver, skeletal muscle and pancreas, we here demonstrate that STK25 also critically controls mitochondrial function, hypertrophy, meta-inflammation and fibrosis progression in BAT and WAT, regulating whole-body insulin sensitization in the context of obesity. It remains to be addressed weather pharmacological inhibition of STK25 is a conceptually viable approach to prevent/treat insulin resistance.

\section{Supplementary data}

This is linked to the online version of the paper at https://doi.org/10.1530/ JOE-18-0182.

\section{Declaration of interest}

The authors declare that there is no conflict of interest that could be perceived as prejudicing the impartiality of the research reported.

\section{Funding}

This work was supported by grants from the Swedish Research Council, the Novo Nordisk Foundation, the Swedish Heart and Lung Foundation, the Diabetes Wellness Network Sweden, the Estonian Research Council, the Swedish Diabetes Foundation, the Royal Society of Arts and Sciences in Gothenburg, the Wiberg Foundation, the Adlerbert Research Foundation, the I. Hultman Foundation, the S. and E. Goljes Foundation, the West Sweden ALF Program, the F. Neubergh Foundation, the I.-B. and A. Lundbergs Research Foundation, the Swedish Innovation Agency Vinnova, the Prof. Nanna Svartz Foundation, the Torsten Söderbergs Foundation, the European Foundation for the Study of Diabetes/ Lilly European Diabetes Research Programme, the L. och J. Grönbergs Foundation, the European Foundation for the Study of Diabetes and Novo Nordisk Partnership for Diabetes Research in Europe and the European Union's Seventh Framework Program (FP7/2007-2013) under grant agreement $n^{\circ} 607842$. The authors declare that there is no duality of interest associated with this manuscript.

\section{Author contribution statement}

All the authors made substantial contributions to conception and design, and/or analysis and interpretation of data. S S, E C, M A, E N-D, N M K and $\mathrm{M} S$ generated the bulk of the results. A $P$ performed the nonlinear microscopy. J L, J B, B W H and S E provided expertise and key reagents and contributed to the discussion. M M directed the project, designed the study, interpreted the data and wrote the manuscript. All the authors revised the article critically for important intellectual content and approved the final version of the article to be published.

\section{Acknowledgements}

The authors acknowledge the technical assistance of Dhirendra Pratap Singh, Elin Magnusson and Annika Nerstedt, Department of Molecular and Clinical Medicine, University of Gothenburg, Sweden, and the Centre for Cellular Imaging at University of Gothenburg and the National Microscopy Infrastructure, NMI (VR-RFI 2016-00968) for providing assistance in microscopy.

\section{References}

Amrutkar M, Cansby E, Chursa U, Nunez-Duran E, Chanclon B, Stahlman M, Friden V, Manneras-Holm L, Wickman A, Smith U, 
et al. $2015 a$ Genetic disruption of protein kinase STK25 ameliorates metabolic defects in a diet-induced type 2 diabetes model. Diabetes 64 2791-2804. (https://doi.org/10.2337/db15-0060)

Amrutkar M, Cansby E, Nunez-Duran E, Pirazzi C, Stahlman M, Stenfeldt E, Smith U, Boren J \& Mahlapuu M 2015b Protein kinase STK25 regulates hepatic lipid partitioning and progression of liver steatosis and NASH. FASEB Journal 29 1564-1576. (https://doi. org/10.1096/fj.14-264937)

Amrutkar M, Chursa U, Kern M, Nunez-Duran E, Stahlman M, Sutt S, Boren J, Johansson BR, Marschall HU, Bluher M, et al. $2016 a$ STK25 is a critical determinant in nonalcoholic steatohepatitis. FASEB Journal 30 3628-3643. (https://doi.org/10.1096/fj.201600562R)

Amrutkar M, Kern M, Nunez-Duran E, Stahlman M, Cansby E, Chursa U, Stenfeldt E, Boren J, Bluher M \& Mahlapuu M $2016 b$ Protein kinase STK25 controls lipid partitioning in hepatocytes and correlates with liver fat content in humans. Diabetologia 59 341-353. (https://doi. org/10.1007/s00125-015-3801-7)

Anderberg C, Cunha SI, Zhai Z, Cortez E, Pardali E, Johnson JR, Franco M, Paez-Ribes M, Cordiner R, Fuxe J, et al. 2013 Deficiency for endoglin in tumor vasculature weakens the endothelial barrier to metastatic dissemination. Journal of Experimental Medicine 210 563-579. (https:// doi.org/10.1084/jem.20120662)

Anstee QM, Targher G \& Day CP 2013 Progression of NAFLD to diabetes mellitus, cardiovascular disease or cirrhosis. Nature Reviews Gastroenterology and Hepatology 10 330-344. (https://doi.org/10.1038/ nrgastro.2013.41)

Aouadi M, Tencerova M, Vangala P, Yawe JC, Nicoloro SM, Amano SU, Cohen JL \& Czech MP 2013 Gene silencing in adipose tissue macrophages regulates whole-body metabolism in obese mice. PNAS 110 8278-8283. (https://doi.org/10.1073/pnas.1300492110)

Arner E, Mejhert N, Kulyte A, Balwierz PJ, Pachkov M, Cormont M, Lorente-Cebrian S, Ehrlund A, Laurencikiene J, Heden P, et al. 2012 Adipose tissue microRNAs as regulators of CCL2 production in human obesity. Diabetes 61 1986-1993. (https://doi.org/10.2337/ db11-1508)

Brannmark C, Paul A, Ribeiro D, Magnusson B, Brolen G, Enejder A \& Forslow A 2014 Increased adipogenesis of human adipose-derived stem cells on polycaprolactone fiber matrices. PLOS ONE 9 e113620. (https://doi.org/10.1371/journal.pone.0113620)

Cansby E, Amrutkar M, Manneras Holm L, Nerstedt A, Reyahi A, Stenfeldt E, Boren J, Carlsson P, Smith U, Zierath JR, et al. 2013 Increased expression of STK25 leads to impaired glucose utilization and insulin sensitivity in mice challenged with a high-fat diet. FASEB Journal 27 3660-3671. (https://doi.org/10.1096/fj.13-228494)

Choo HJ, Kim JH, Kwon OB, Lee CS, Mun JY, Han SS, Yoon YS, Yoon G, Choi KM \& Ko YG 2006 Mitochondria are impaired in the adipocytes of type 2 diabetic mice. Diabetologia 49 784-791. (https://doi. org/10.1007/s00125-006-0170-2)

Chursa U, Nunez-Duran E, Cansby E, Amrutkar M, Sutt S, Stahlman M, Olsson BM, Boren J, Johansson ME, Backhed F, et al. 2017 Overexpression of protein kinase STK25 in mice exacerbates ectopic lipid accumulation, mitochondrial dysfunction and insulin resistance in skeletal muscle. Diabetologia 60 553-567. (https://doi.org/10.1007/ s00125-016-4171-5)

Egan JJ, Greenberg AS, Chang MK, Wek SA, Moos MC Jr \& Londos C 1992 Mechanism of hormone-stimulated lipolysis in adipocytes: translocation of hormone-sensitive lipase to the lipid storage droplet. PNAS 89 8537-8541. (https://doi.org/10.1073/pnas.89.18.8537)

Evans CL \& Xie XS 2008 Coherent anti-stokes Raman scattering microscopy: chemical imaging for biology and medicine. Annual Review of Analytical Chemistry 1 883-909. (https://doi.org/10.1146/ annurev.anchem.1.031207.112754)

Fujimori K \& Amano F 2011 Niacin promotes adipogenesis by reducing production of anti-adipogenic PGF2alpha through suppression of C/EBPbeta-activated COX-2 expression. Prostaglandins and
Other Lipid Mediators 94 96-103. (https://doi.org/10.1016/j. prostaglandins.2011.01.002)

Geerling JJ, Boon MR, van der Zon GC, van den Berg SA, van den Hoek AM, Lombes M, Princen HM, Havekes LM, Rensen PC \& Guigas B 2014 Metformin lowers plasma triglycerides by promoting VLDL-triglyceride clearance by brown adipose tissue in mice. Diabetes 63 880-891. (https://doi.org/10.2337/db13-0194)

Gustafson B \& Smith U 2006 Cytokines promote Wnt signaling and inflammation and impair the normal differentiation and lipid accumulation in 3T3-L1 preadipocytes. Journal of Biological Chemistry 281 9507-9516. (https://doi.org/10.1074/jbc.M512077200)

Harms M \& Seale P 2013 Brown and beige fat: development, function and therapeutic potential. Nature Medicine 19 1252-1263. (https://doi. org/10.1038/nm.3361)

Heinonen S, Buzkova J, Muniandy M, Kaksonen R, Ollikainen M, Ismail K, Hakkarainen A, Lundbom J, Lundbom N, Vuolteenaho K, et al. 2015 Impaired mitochondrial biogenesis in adipose tissue in acquired obesity. Diabetes 64 3135-3145. (https://doi.org/10.2337/ db14-1937)

Homan R \& Anderson MK 1998 Rapid separation and quantitation of combined neutral and polar lipid classes by high-performance liquid chromatography and evaporative light-scattering mass detection. Journal of Chromatography B Biomedical Sciences and Applications $\mathbf{7 0 8}$ 21-26. (https://doi.org/10.1016/S0378-4347(97)00651-8)

Kusminski CM, Bickel PE \& Scherer PE 2016 Targeting adipose tissue in the treatment of obesity-associated diabetes. Nature Reviews Drug Discovery 15 639-660. (https://doi.org/10.1038/nrd.2016.75)

Lofgren L, Forsberg GB \& Stahlman M 2016 The BUME method: a new rapid and simple chloroform-free method for total lipid extraction of animal tissue. Scientific Reports 6 27688. (https://doi.org/10.1038/ srep27688)

Lumeng CN \& Saltiel AR 2011 Inflammatory links between obesity and metabolic disease. Journal of Clinical Investigation $1212111-2117$. (https://doi.org/10.1172/JCI57132)

Matsuki T, Chen J \& Howell BW 2013 Acute inactivation of the serine-threonine kinase Stk25 disrupts neuronal migration. Neural Development 8 21. (https://doi.org/10.1186/1749-8104-8-21)

Mouras R, Bagnaninchi P, Downes A, Muratore M \& Elfick A 2011 Non linear optical microscopy of adipose-derived stem cells induced towards osteoblasts and adipocytes. Proceedings of SPIE: Advanced Microscopy Techniques II 8086 8086Q. (https://doi. org/10.1117/12.889780)

Nedergaard J \& Cannon B 2013 UCP1 mRNA does not produce heat. Biochimica et Biophysica Acta 1831 943-949. (https://doi. org/10.1016/j.bbalip.2013.01.009)

Nedergaard J, Bengtsson T \& Cannon B 2010 Three years with adult human brown adipose tissue. Annals of the New York Academy of Sciences 1212 E20-E36. (https://doi.org/10.1111/j.17496632.2010.05905.x)

Nerstedt A, Cansby E, Andersson CX, Laakso M, Stancakova A, Bluher M, Smith U \& Mahlapuu M 2012 Serine/threonine protein kinase 25 (STK25): a novel negative regulator of lipid and glucose metabolism in rodent and human skeletal muscle. Diabetologia 55 1797-1807. (https://doi.org/10.1007/s00125-012-2511-7)

Nunez-Duran E, Chanclon B, Sutt S, Real J, Marschall HU, Wernstedt Asterholm I, Cansby E \& Mahlapuu M 2017 Protein kinase STK25 aggravates the severity of non-alcoholic fatty pancreas disease in mice. Journal of Endocrinology 234 15-27. (https://doi.org/10.1530/ JOE-17-0018)

Nunez-Duran E, Aghajan M, Amrutkar M, Sütt S, Cansby E, Booten SL, Watt A, Ståhlman M, Stefan N, Häring H-U, et al. 2018 Serine/ threonine protein kinase 25 antisense oligonucleotide treatment reverses glucose intolerance, insulin resistance and nonalcoholic fatty liver disease in mice. Hepatology Communications 2 69-83. (https://doi. org/10.1002/hep4.1128) 
Pasarica M, Gowronska-Kozak B, Burk D, Remedios I, Hymel D, Gimble J, Ravussin E, Bray GA \& Smith SR 2009 Adipose tissue collagen VI in obesity. Journal of Clinical Endocrinology and Metabolism 94 5155-5162. (https://doi.org/10.1210/jc.2009-0947)

Reid BN, Ables GP, Otlivanchik OA, Schoiswohl G, Zechner R, Blaner WS Goldberg IJ, Schwabe RF, Chua SC Jr \& Huang LS 2008 Hepatic overexpression of hormone-sensitive lipase and adipose triglyceride lipase promotes fatty acid oxidation, stimulates direct release of free fatty acids, and ameliorates steatosis. Journal of Biological Chemistry 283 13087-13099. (https://doi.org/10.1074/jbc.M800533200)

Rong JX, Qiu Y, Hansen MK, Zhu L, Zhang V, Xie M, Okamoto Y, Mattie MD, Higashiyama H, Asano S, et al. 2007 Adipose mitochondrial biogenesis is suppressed in $\mathrm{db} / \mathrm{db}$ and high-fat diet-fed mice and improved by rosiglitazone. Diabetes 56 1751-1760. (https:// doi.org/10.2337/db06-1135)

Rosen E \& Spiegelman B 2014 What we talk about when we talk about fat. Cell 16 20-44. (https://doi.org/10.1016/j.cell.2013.12.012)

Savage DB, Choi CS, Samuel VT, Liu ZX, Zhang D, Wang A, Zhang XM, Cline GW, Yu XX, Geisler JG, et al. 2006 Reversal of diet-induced hepatic steatosis and hepatic insulin resistance by antisense oligonucleotide inhibitors of acetyl-CoA carboxylases 1 and 2. Journal of Clinical Investigation 116 817-824. (https://doi.org/10.1172/JCI27300)

Sjostrom L, Gummesson A, Sjostrom CD, Narbro K, Peltonen M, Wedel H, Bengtsson C, Bouchard C, Carlsson B, Dahlgren S, et al. 2009 Effects of bariatric surgery on cancer incidence in obese patients in Sweden (Swedish Obese Subjects Study): a prospective, controlled intervention trial. Lancet Oncology 10 653-662. (https://doi.org/10.1016/S14702045(09)70159-7)

Spencer M, Yao-Borengasser A, Unal R, Rasouli N, Gurley CM, Zhu B, Peterson CA \& Kern PA 2010 Adipose tissue macrophages in insulinresistant subjects are associated with collagen VI and fibrosis and demonstrate alternative activation. American Journal of Physiology: Endocrinology and Metabolism 299 E1016-E1027. (https://doi. org/10.1152/ajpendo.00329.2010)

Sun K, Tordjman J, Clement K \& Scherer PE 2013 Fibrosis and adipose tissue dysfunction. Cell Metabolism 18 470-477. (https://doi. org/10.1016/j.cmet.2013.06.016)

Sunny NE, Bril F \& Cusi K 2017 Mitochondrial adaptation in nonalcoholic fatty liver disease: novel mechanisms and treatment strategies. Trends in Endocrinology and Metabolism 28 250-260. (https:// doi.org/10.1016/j.tem.2016.11.006)

Thompson BJ \& Sahai E 2015 MST kinases in development and disease. Journal of Cell Biology 210 871-882. (https://doi.org/10.1083/ jcb.201507005)

Tunaru S, Kero J, Schaub A, Wufka C, Blaukat A, Pfeffer K \& Offermanns S 2003 PUMA-G and HM74 are receptors for nicotinic acid and mediate its anti-lipolytic effect. Nature Medicine 9 352-355. (https://doi. org/10.1038/nm824)

Van Gaal LF, Mertens IL \& De Block CE 2006 Mechanisms linking obesity with cardiovascular disease. Nature 444 875-880. (https://doi. org/10.1038/nature05487)

Wang M \& Kaufman RJ 2016 Protein misfolding in the endoplasmic reticulum as a conduit to human disease. Nature 529 326-335. (https://doi.org/10.1038/nature17041)

Vernochet C, Peres SB, Davis KE, McDonald ME, Qiang L, Wang H, Scherer PE \& Farmer SR 2009 C/EBPalpha and the corepressors CtBP1 and CtBP2 regulate repression of select visceral white adipose genes during induction of the brown phenotype in white adipocytes by peroxisome proliferator-activated receptor gamma agonists. Molecular and Cellular Biology 29 4714-4728. (https://doi.org/10.1128/ MCB.01899-08)

Vernochet C, Mourier A, Bezy O, Macotela Y, Boucher J, Rardin MJ, An D, Lee KY, Ilkayeva OR, Zingaretti CM, et al. 2012 Adipose-specific deletion of TFAM increases mitochondrial oxidation and protects mice against obesity and insulin resistance. Cell Metabolism 16 765-776. (https://doi.org/10.1016/j.cmet.2012.10.016)

Xu H, Barnes GT, Yang Q, Tan G, Yang D, Chou CJ, Sole J, Nichols A Ross JS, Tartaglia LA, et al. 2003 Chronic inflammation in fat plays a crucial role in the development of obesity-related insulin resistance. Journal of Clinical Investigation 112 1821-1830. (https://doi. org/10.1172/JCI200319451)

Zhang K \& Kaufman RJ 2008 From endoplasmic-reticulum stress to the inflammatory response. Nature $\mathbf{4 5 4} 455-462$. (https://doi. org/10.1038/nature07203)

Zumbusch A, Holtom GR \& Xie XS 1999 Three-dimensional vibrational imaging by coherent anti-stokes Raman scattering. Physical Review Letters 82 4142-4145. (https://doi.org/10.1103/ PhysRevLett.82.4142)

Received in final form 7 May 2018

Accepted 24 May 2018

Accepted Preprint published online 24 May 2018 (c) 2018 Society for Endocrinology Published by Bioscientifica Ltd. Printed in Great Britain 